\title{
Non-equilibrium quadratic measurement-feedback squeezing in a micromechanical resonator
}

\author{
Motoki Asano, ${ }^{1}$ Takuma Aihara, ${ }^{2}$ Tai Tsuchizawa, ${ }^{2}$ and Hiroshi Yamaguchi ${ }^{1}$ \\ ${ }^{1}$ NTT Basic Research Laboratories, NTT Corporation, 3-1 Morinosato Wakamiya, Atsugi-shi, Kanagawa 243-0198, Japan \\ ${ }^{2}$ NTT Device Technology Laboratories, NTT Corporation, 3-1 Morinosato Wakamiya, Atsugi-shi, Kanagawa 243-0198, Japan
}

(Received 6 April 2021; revised 16 June 2021; accepted 29 June 2021; published 6 August 2021)

\begin{abstract}
Measurement and feedback control of thermomechanical motion in a micromechanical resonator has been actively studied to achieve extremely high sensing performance by controlling the stochastic thermal noise. Although linear measurement-feedback control in the phase space results in the feedback cooling, extending them to the nonlinear regime, i.e., utilizing quadratic of higher-order dynamic variables in both measurement and control, can further functionalize its operations. Here, we demonstrate fully quadratic measurement-feedback protocol in a micromechanical resonator by driving the second-order nonlinearity and directly measuring quadratic variables referred as Schwinger angular momentum. Our measurement-feedback protocol enables us to achieve a noise reduction at the level of $-5.1 \pm 0.2 \mathrm{~dB}$ over the $-3-\mathrm{dB}$ limitation in the continuous parametric driving. We unveil that this strong noise reduction originates in the effective cooling effect by investigating entropy production rates. These results would be further extended to investigating general performance of nonlinear information thermodynamic machines, in which the higher-order moments (e.g., variance and correlations) can be controlled with avoiding the nonlinear instability, thanks to the existence of information flows.
\end{abstract}

DOI: 10.1103/PhysRevResearch.3.033121

\section{INTRODUCTION}

Measurement-feedback control of fluctuation in mesoscopic systems has attracted large interest to investigate stochastic dynamics under measurement and feedback. The presences of a measurement-feedback controller modifies the balance of entropy flows (i.e., the second law in the total system), and allows us to extract finite work to effectively heat up or cool down the system [1]. Such stochastic thermodynamics under the measurement and feedback have been investigated in various types of mesoscopic systems with artificial systems as well as natural biological ones [2]. In particular, micromechanical systems, such as an optically trapped nanoparticles [3-5] and micro/nanomechanical resonators [6-9], have been widely used to investigate the stochastic thermodynamics because fluctuation of their displacement (or velocity) can be precisely detected and controlled to implement measurementfeedback protocols.

So far, the linear measurement-feedback protocols, which consists of measurement and feedback of linear variables, have been demonstrated in micromechanical resonators to reduce the effective temperature in random motion [10-12]. The cooling mechanism in their linear measurement-feedback control has been unveiled in both theory $[13,14]$ and experiment [15] in terms of entropy production. This linear feedback cooling has been embedded in the noise squeez-

Published by the American Physical Society under the terms of the Creative Commons Attribution 4.0 International license. Further distribution of this work must maintain attribution to the author(s) and the published article's title, journal citation, and DOI. ing schemes [16,17], which resulted in the stronger noise reduction than the no-cooling case. As a natural but important extension to general stochastic dynamics, bringing nonlinear variables to the measurement and feedback can further investigate and functionalize its thermodynamic costs in information flows generalized by the map from the linear phase space to the nonlinear variable space. Although micro/nanomechanical resonators have been individually used to investigate both nonlinear control [18-20] and nonlinear measurement [21-23], combining them to develop a nonlinear measurement-feedback protocol has not been reported yet.

In this paper, we propose and demonstrate continuous measurement-feedback control of a micromechanical resonator based on a quadratic variable, which is referred as "Schwinger angular momentum" because it is the quadratic form defined in the angular-momentum representation of bosons [24]. Because the Schwinger angular momentum holds quadratic and symmetric properties with $\mathrm{SU}(1,1)$ Lie algebra, we develop a continuous measurement-feedback protocol by combining parametric nonlinearity and measurement nonlinearity. The measurement nonlinearity enables us to directly readout the component of Schwinger angular momentum via nonlinear optomechanical transduction. The parametric nonlinearity enables us to drive the Schwinger angular momentum and squeeze the noise deviation (i.e., noise compression along a quadrature and noise amplification along an orthogonal one). In contrast to the randomly pulsed parametric driving without measurement, our continuous measurement-feedback protocol enables us to achieve a non-equilibrium steady state (NESS) with strong noise squeezing at the level of $-5.1 \pm$ $0.2 \mathrm{~dB}$. This is because the quadratic measurement-feedback protocol involves a net cooling effect so that it avoids the 
(a)
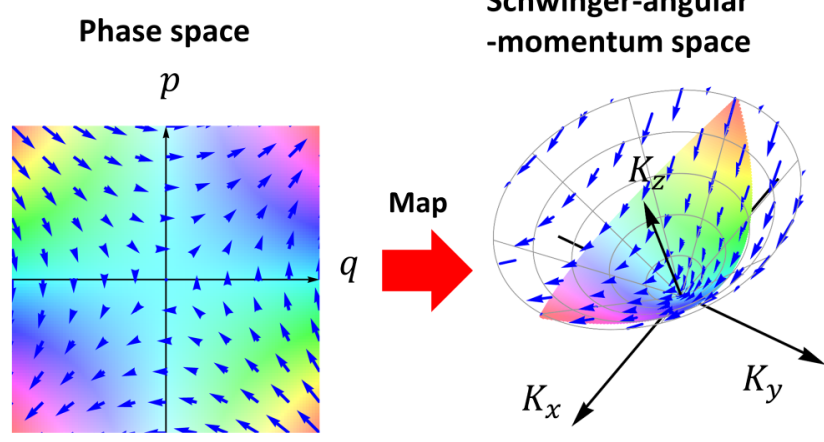

(b)

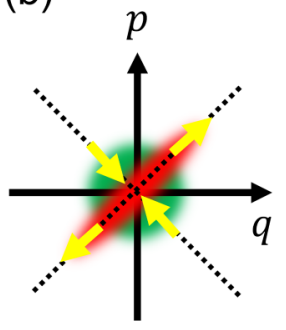

(c)

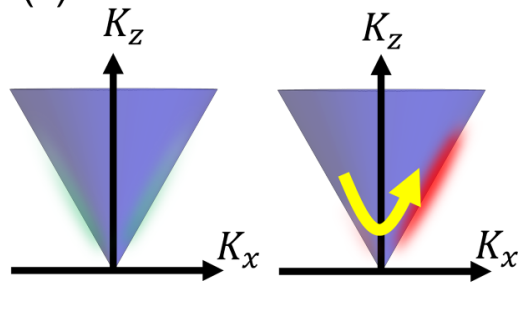

FIG. 1. (a) Conceptual illustration of the phase space spanned by $q$ and $p$, and the Schwinger angular-momentum space spanned by $K_{x}, K_{y}$, and $K_{z}$. The color map shows correspondence between the phase space and the Schwinger angular-momentum space. The blue vectors show the force field of the parametric squeezing with $\mathcal{H}_{\text {eff }}$. [(b),(c)] Schematic of probability distribution of thermal equilibrium (green) and steady state with continuous squeezing (red) in the phase space and the Schwinger angular-momentum space, respectively.

divergence in parametric squeezing, which limits the level at $-3 \mathrm{~dB}$. This net cooling effect is unveiled by investigating the entropy production rates in our measurement-feedback protocols with a coarse-grained model.

\section{PROTOCOL}

The basic idea is to map the dynamic variables represented by $(q, p)$ in the rotating-frame phase space into the Schwinger angular-momentum space spanned by the following three components:

$$
K_{x}=\frac{q p}{2}, \quad K_{y}=\frac{q^{2}-p^{2}}{4}, \quad K_{z}=\frac{q^{2}+p^{2}}{4},
$$

where these components satisfy $K_{x}^{2}+K_{y}^{2}-K_{z}^{2}=0$ (i.e., the dynamics is constrained on a hyperboloid) [see Fig. 1(a)]. In addition to the geometrical property, they also satisfy a Lie algebra in $\mathrm{SU}(1,1)$ group such that

$$
\left\{K_{x}, K_{y}\right\}=-K_{z}, \quad\left\{K_{y}, K_{z}\right\}=K_{x}, \quad\left\{K_{z}, K_{x}\right\}=K_{y},
$$

where $\{A, B\}$ denotes the Poisson bracket defined by $\{A, B\} \equiv$ $\partial A / \partial q \partial B / \partial p-\partial A / \partial p \partial B / \partial q$. Since the relation $\left\{K_{x}, K_{y}\right\}=$ $-K_{z}$ only contains a negative sign compared with the other two, the effective Hamiltonian, $\mathcal{H}_{\text {eff }}=-G_{0} K_{y}$, leads a pseudorotation around the $K_{y}$ axis in the Schwinger angularmomentum space [see arrows in Fig. 1(a)]. Importantly, this pseudorotation Hamiltonian is obtained by driving the twice of mechanical frequency via parametric nonlinearity (see Appendix A). Thus, the parametric driving induces such a pseudorotation dynamics given by

$$
\dot{K}_{x}=G_{0} K_{z}, \quad \dot{K}_{z}=G_{0} K_{x}, \quad \dot{K}_{y}=0 .
$$

Apparently, the general solutions of $K_{x}$ and $K_{z}$ are given by hyperbolic sine and cosine functions of time, which describes the dynamics of the parametric squeezing under the continuous driving [18].

The above formulation is valid to describe the stochastic dynamics of mechanical resonators with a probability density function $\mathcal{P}(q, p)$ in its phase space and $\mathcal{P}\left(K_{x}, K_{y}, K_{z}\right)$ in the Schwinger angular-momentum space. In the phase-space description, the parametric squeezing with $\mathcal{H}_{\text {eff }}$ amplifies the deviation along a diagonal quadrature, $q_{+} \equiv(q+p) / \sqrt{2}$, and reduces the deviation along its orthogonal portion, $q_{-} \equiv(q-$ p) $/ \sqrt{2}$ direction [see Fig. 1(b)]. On the other hand, in the Schwinger angular-momentum description, the pseudorotation with $\mathcal{H}_{\text {eff }}$ around the $K_{y}$ axis leads to a biased probability distribution along the $K_{z}+K_{x}$ direction [see Fig. 1(c)]. Importantly, this pseudorotation can be decomposed into noise compression along $K_{z}-K_{x}$ and noise expansion along $K_{z}+$ $K_{x}$. By taking into account that

$$
\left\langle K_{z} \pm K_{x}\right\rangle=\sigma\left(q_{ \pm}\right)^{2} / 2,
$$

with the standard deviation of $q_{ \pm}, \sigma\left(q_{ \pm}\right)$, compression (expansion) along the $K_{z}-K_{x}\left(K_{z}+K_{x}\right)$ is regarded as the noise reduction (amplification) in the phase space. The divergence, which intrinsically limits the noise reduction level at $-3 \mathrm{~dB}$ [18], appears as an infinite large noise amplification (i.e., noise expansion along $K_{z}+K_{x}$ direction in the pseudorotation) when the drive strength $G_{0}$ is equivalent to the mechanical damping factor $\Gamma$.

Because the divergent contribution in parametric drive can be distinguished with the sign of $K_{x}$, to avoid the divergence, our measurement-feedback protocol is derived as a switching operation of the parametric drive with respect to this sign. Switching on the parametric drive (i.e., $G_{0}$ takes a nonzero value) only when $K_{x}<0$ leads a suppression of the probability density function in the Schwinger angular-momentum space. On the other hand, switching it off $\left(G_{0}=0\right)$ when $K_{x}>0$ can avoid the divergence in parametric driving. To read out the sign of $K_{x}$ to construct a feedback loop, we can utilize nonlinear optomechanical transduction in which higher harmonic signals are generated thanks to a dispersive modulation of optical phase via mechanical motion [21-23]. In particular, the sine and cosine parts in the second-order harmonics are regarded as $K_{x}$ and $K_{y}$, respectively. To achieve steady-state squeezing, our measurement-feedback protocol is continuously repeated with $K_{x}$ directly measured via nonlinear optomechanical transduction and the parametric drive switched on or off with respect to the sign of measured $K_{x}$ [see Fig. 2(a)].

Although the parametric squeezing under the continuous driving heats up the system (see Appendix B), we can expect that avoiding the heating part of pseudorotation [i.e., $K_{x}>0$ in Fig. 1(c)] in Schwinger angular-momentum space with our measurement-feedback protocol effectively induces a net cooling effect. To unveil this net cooling effect hidden in our protocol, we take into account entropy production in our 
(a)

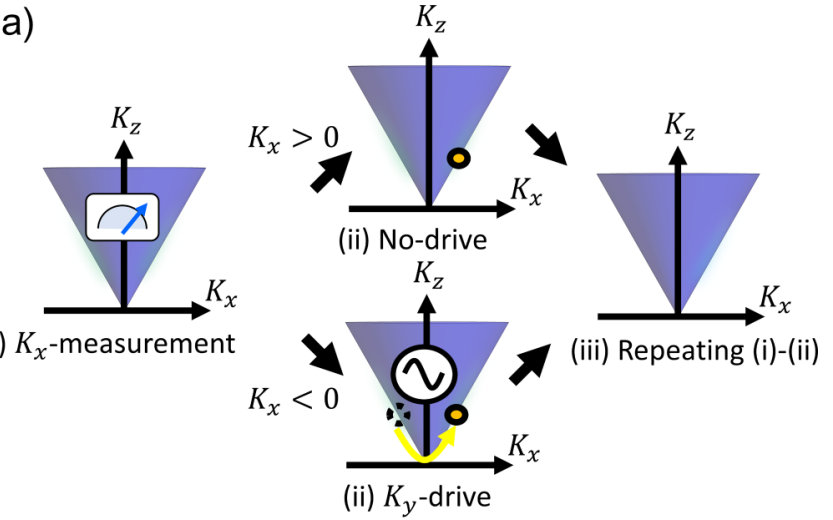

(b)

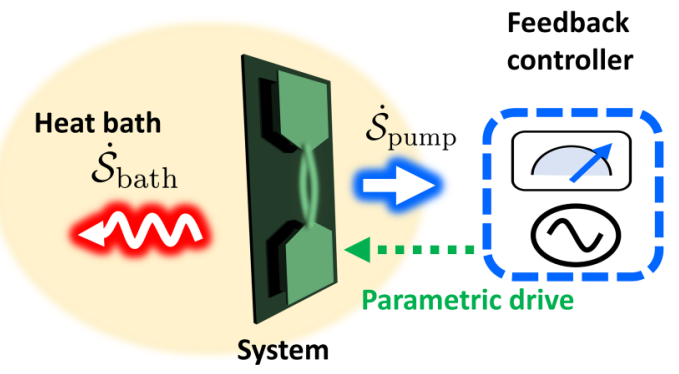

FIG. 2. (a) Schematic of the measurement-feedback protocol in the Schwinger angular-momentum space. Initially, the sign of $K_{x}$ is measured via nonlinear optomechanical transduction (i). Then, only if $K_{x}<0$, the parametric drive is switched on (ii). By repeating these two processes, we achieve a non-equilibrium steady state (NESS) that is partially distributed around only $K_{x}>0$ (iii). (b) Conceptual illustration of net cooling via measurement-feedback protocol. In addition to the entropy production in the thermal bath with its rate $\dot{\mathcal{S}}_{\text {bath }}$, there exists entropy production called "entropy pumping" with the rate $\dot{\mathcal{S}}_{\text {pump }}$ due to the existence of the feedback controller. The vectors describe the actual directions of both fluxes when the measurementfeedback protocol succeeds, where $\dot{\mathcal{S}}_{\text {bath }} \geqslant 0$ and $\dot{\mathcal{S}}_{\text {pump }} \geqslant 0$ in our definition.

setup, which consists of a system (a mechanical resonator), a heat bath, and a feedback controller [see Fig. 2(b)] by a coarse-graining methods, where the degree of feedback memory is coarse-grained $[13,14,25]$. The total entropy production rate consists of two contributions: the entropy production in the thermal bath $\dot{\mathcal{S}}_{\text {bath }}$, and the entropy production thanks to the existence of the feedback controller $\dot{\mathcal{S}}_{\text {pump. }}$. The former corresponds to the heat flux between the system and the heat bath, and the latter corresponds to the entropy flow pumped from the feedback controller. They can be expressed in terms of the Schwinger angular momentum as follows:

$$
\begin{gathered}
\left\langle\dot{\mathcal{S}}_{\text {bath }}\right\rangle \approx \frac{2 \Omega^{2} \Gamma}{k_{B} T}\left(\left\langle K_{z}\right\rangle-\frac{k_{B} T}{2 \Omega^{2}}\right), \\
\left\langle\dot{\mathcal{S}}_{\text {pump }}\right\rangle \approx \sqrt{\frac{1}{2 \pi}} \frac{\tilde{G}_{0} \Gamma}{\sigma_{M}}\left\langle K_{z} \exp \left(-\frac{K_{x}^{2}}{2 \sigma_{M}^{2}}\right)\right\rangle,
\end{gathered}
$$

where $\langle\cdot\rangle$ denotes the stochastic average, and $\tilde{G}_{0}=G_{0} / \Gamma$ is the effective driving strength as a dimensionless quantity. The detailed derivation of Eqs. (5) and (6) are shown in both Appendices $\mathrm{C}$ and $\mathrm{D}$, where the path-integral formalism $[13,25,26]$ and probability-current formalism $[14,27,28]$ are utilized, respectively. The entropy production in the thermal bath is given by the shift of the vertical component of Schwinger angular momentum $K_{z}$ from its value in the equilibrium because $K_{z}$ directly corresponds to the oscillation energy (phonon number) in the resonator. On the other hand, the entropy pumping rate is given by a function of $K_{x}, K_{z}$, and the measurement noise characterized by its deviation of $\sigma_{M}$. Because of $K_{z} \geqslant 0,\left\langle\dot{\mathcal{S}}_{\text {pump }}\right\rangle \geqslant 0$ always holds, which indicates that the entropy pumping operates to pull the entropy from the system. These three contributions satisfy the secondlaw-like inequality

$$
\left\langle\dot{\mathcal{S}}_{\text {bath }}\right\rangle+\left\langle\dot{\mathcal{S}}_{\text {pump }}\right\rangle \geqslant 0 .
$$

This inequality implies that $\left\langle\dot{\mathcal{S}}_{\text {bath }}\right\rangle$ may take a negative value in contrast to the case of no-feedback operation where $\left\langle\dot{\mathcal{S}}_{\text {bath }}\right\rangle \geqslant 0$ holds. Note that $\left\langle\dot{\mathcal{S}}_{\text {bath }}\right\rangle \geqslant 0$ holds when the heat flux flows out from the system to the heat bath, and $\left\langle\dot{\mathcal{S}}_{\text {pump }}\right\rangle \geqslant$ 0 holds when the feedback controller pumps out the entropy from the system. Thus, this inequality implies that the system may operate as a cooler by pumping the heat flux from the heat bath to the feedback controller. Although it is hard to directly define the effective temperature of system due to its nonGaussian distribution in the NESS, we can define the heating regime with $\left\langle\dot{\mathcal{S}}_{\text {bath }}\right\rangle>0$, where the heat flux flows out from the system to the bath, and the cooling regime with $\left\langle\dot{\mathcal{S}}_{\text {bath }}\right\rangle<$ 0 , where the heat flux flows in the system from the bath.

\section{EXPERIMENT}

\section{A. Setup}

Our measurement-feedback protocol was implemented on a measurement-feedback loop with a micromechanical resonator [see Fig. 3(a)]. A doubly-clamped silicon nitride mechanical resonator $(150-\mu \mathrm{m}$-long, $5-\mu \mathrm{m}$-wide, and 525 -nm-thick) was fabricated via thermal chemical vapor deposition, and placed in a vacuum environment $\left(\sim 10^{-4} \mathrm{~Pa}\right)$. The resonator showed a high quality factor of $3.0 \times 10^{4}$ in its fundamental flexural mode at the frequency of $\Omega=$ $2 \pi \times 510 \mathrm{kHz}$ at room temperature. Linear quadratures $(q$ and $p$ ) and a component of Schwinger angular momentum $\left(K_{x}^{M}\right)$ in its mechanical motion were extracted from a laser Doppler interferometer (LDI). Here, we denote the directly measured Schwinger angular-momentum component with $K_{x}^{M}$ to distinguish it from the one, $K_{x}^{P}=q p / 2$, calculated via postprocessing with the measured $q$ and $p$. The output of LDI was connected to lock-in amplifiers with a reference frequency of $\Omega$ for the linear quadratures and that of $2 \Omega$ for the Schwinger angular-momentum components. Note that we induced additional white noise via an piezoelectric sheet attached on the resonator substrate to improve the signal-tonoise ratio for $K_{x}^{M}$ (the effective temperature was estimated to be $T_{\text {eff }} \approx 10^{5} \mathrm{~K}$ ). By once connecting the output from LDI to a spectrum analyzer, spectrum at $2 \Omega$, which reflects the signal of $K_{x}^{M}$, was observed as well as that at $\Omega$ [see Fig. 3(b)]. The linewidth at $2 \Omega$ becomes twice of that at $\Omega$ because $K_{x}^{M}$ is the quadratic variable in $q$ and $p$. The measured component $K_{x}^{M}$ was used to switch on (off) the parametric pump with an oscillation frequency of $2 \Omega$ when the component $K_{x}^{M}$ is negative (positive) with a radio-frequency switch. To pump the 

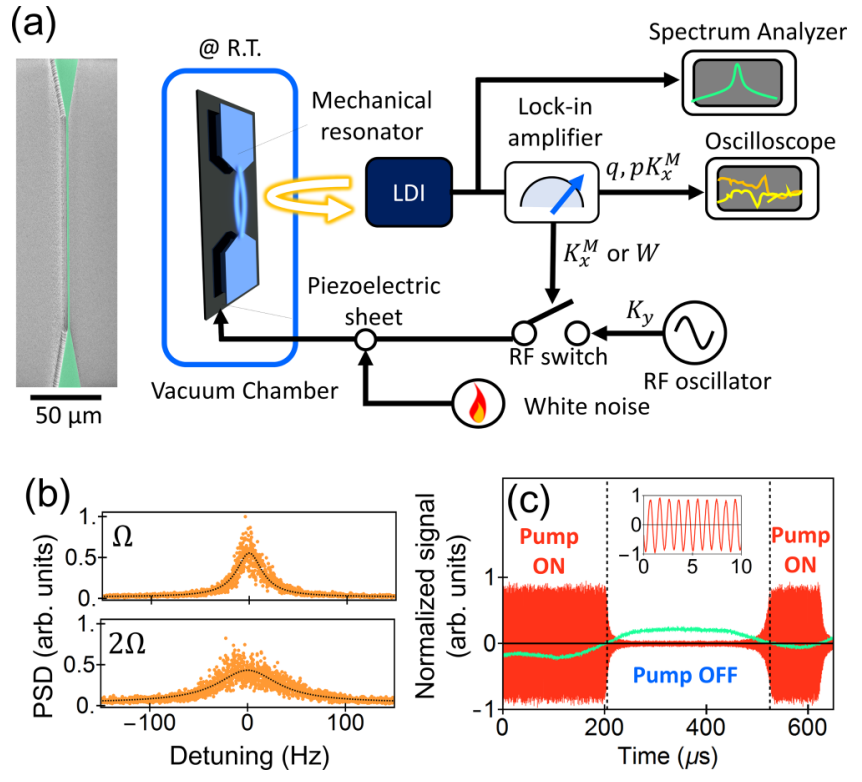

FIG. 3. (a) Schematic of experimental setup with a high-Q silicon nitride doubly-clamped beam. A radio-frequency (rf) oscillator with twice of the mechanical frequency was connected to an rf switch for the parametric driving. This switching operation was determined with respect to the measurement outcome of $K_{x}^{M}$ from a laser Doppler interferometer (LDI). The mechanical excitation was done by the parametric drive signal from the rf oscillator and white noise signal via a piezoelectric signals. Here, note that this interferometer also yields temporal data of the phase quadratures $q$ and $p$ recorded on the oscilloscope. (b) Frequency spectra at $\Omega$ (measurement of phase quadratures) and $2 \Omega$ (measurement of Schwinger angular momenta). (c) Typical temporal sequence of our measurement-feedback protocol. The parametric drive signal with $2 \Omega$ frequency (red curve) was sent to the piezoelectric sheet only if the measurement outcome (the signal of $K_{x}^{M}$ ) took negative values. The inset shows the enlarged data which explicitly indicate the period of $1 /(2 \Omega)$.

mechanical resonator along the $K_{y}$ direction, the phase of the parametric pump was 90-degrees shifted from that for the reference signal to the lock-in amplifier for measuring $K_{x}^{M}$. The parametric pump was feedback to the mechanical resonator via the piezoelectric sheet. A typical temporal response in this measurement-feedback loop is shown in Fig. 3(c) where the parametric pump (red curve) with $2 \Omega$ was turned on when $K_{x}^{M}$ (green plot) was negative. Note that the time constant of the lock-in amplifier was fixed at $\tau_{L}=100 \mu \mathrm{sec}$ to achieve all information on mechanical motion with the mechanical lifetime $\tau_{M}=59 \mathrm{msec}$.

\section{B. Noise reduction level and NESS in Schwinger angular-momentum space}

An initial equilibrium probability distribution was observed without any feedback drive as an equally-distributed Gaussian distribution. To verify how our protocol affects the probability distribution, we demonstrated both random pumping (i.e., the parametric drive was randomly switched on or off) and our measurement-feedback protocol, and evaluated noise reduction and amplification levels in their non-equilibrium steady states. Here, the noise reduction level
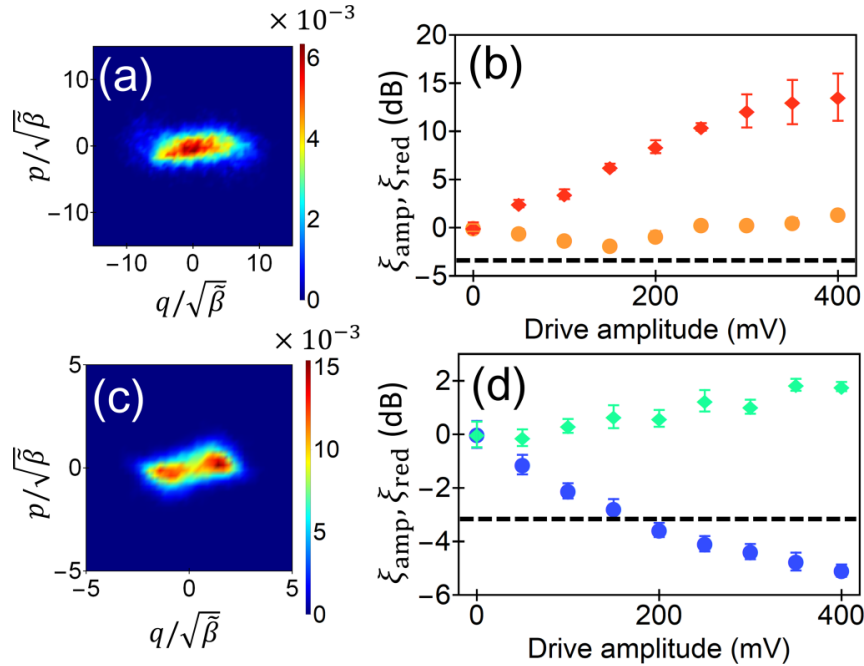

FIG. 4. [(a),(c)] Probability density functions in the phase space with the random protocol and our measurement-feedback protocol, respectively. [(b),(d)] The maximum and minimum standard deviations of these distributions are evaluated with respect to the parametric drive amplitudes The vertical error-bar corresponds to the standard deviation in ten trials.

$\xi_{\text {red }}$ is defined as $\min _{\theta} \sigma\left(q_{\theta, \mathrm{NESS}}\right) / \sigma\left(q_{\mathrm{INIT}}\right)$, where $\sigma(\cdot)$ is the standard deviation, $q_{\text {INIT }}$ is the quadrature in the initial equilibrium, and $q_{\theta}=q \cos \theta+p \sin \theta$ is the quadrature with an arbitrary angle $\theta$. The noise amplification level $\xi_{\text {amp }}$ is defined as the deviation along the orthogonal part, i.e., $\min _{\theta} \sigma\left(\bar{q}_{\theta, \mathrm{NESS}}\right) / \sigma\left(q_{\mathrm{INIT}}\right)$, where $\bar{q}_{\theta}=-q \sin \theta+p \cos \theta$. In the case of the random pumping [see Fig. 4(a)], a squeezed Gaussian distribution with $\xi_{\text {red }} \leqslant \xi_{\text {amp }}$ was achieved in the same way as in standard noise squeezing. However, the noise reduction level was limited to about $-3 \mathrm{~dB}$ around the drive voltage of $150 \mathrm{mV}$ [see Fig. 4(b)]. Drive voltages larger than $150 \mathrm{mV}$ induced parametric instability where both $\xi_{\text {red }}$ and $\xi_{\text {amp }}$ increased. On the other hand, once our measurement-feedback protocol was demonstrated, a nonequilibrium steady state with $\xi_{\text {red }} \leqslant \xi_{\text {amp }}$ was observed with a non-Gaussian probability distribution [see Fig. 4(c)]. This non-Gaussianity directly reflects the non-Gaussian properties of our quadratic variables [29]. Moreover, the noise reduction level finally reached $-5.1 \pm 0.2 \mathrm{~dB}$ over the -3 - $\mathrm{dB}$ limit, whereas the noise amplification level was suppressed at $1.7 \pm$ $0.2 \mathrm{~dB}$ [see Fig. 4(d)]. Importantly, our feedback protocol has an intrinsic lower bound about the noise reduction level. Although it is hard to analytically derive the bound due to the nonlinearity in its dynamics, this bound is confirmed in the numerical calculation shown in Appendix E.

Because we attempt to perform fully quadratic measurement-feedback protocol on the Schwinger angularmomentum space, the NESS in that space is totally different between our measurement-feedback protocol and the random protocol. In contrast to $K_{x}^{M}$, we use the data set of $q$ and $p$ to evaluate Schwinger angular momentum $K_{i}^{P}(i=x, y, z)$ via the postprocessing to reconstruct the probability density function in the Schwinger angular-momentum space. Note that $K_{i}^{P}$ has a more accurate value than $K_{i}^{M}$ because the signal-to-noise ratio in $\Omega$ is better than that in $2 \Omega$, although 


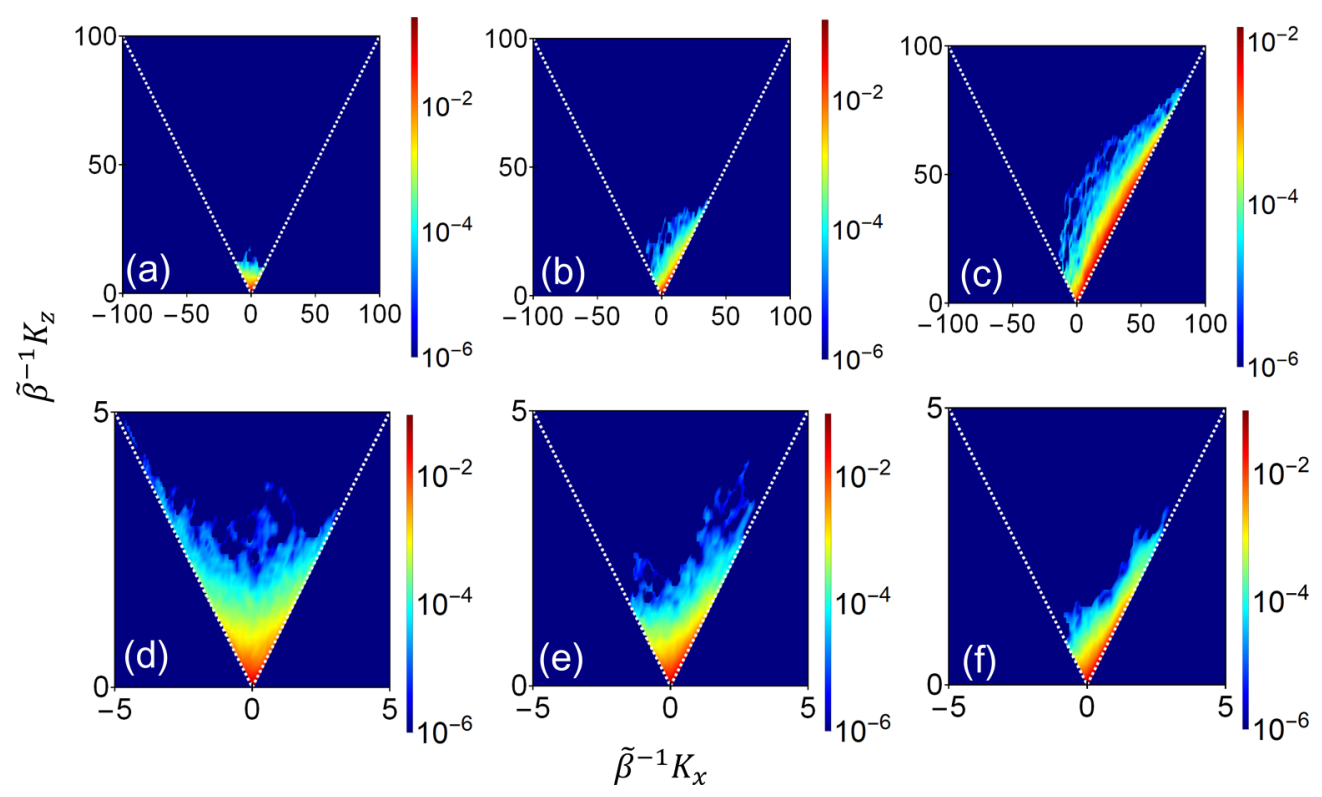

FIG. 5. Probability density functions with the random protocol with the drive amplitudes of (a) $0 \mathrm{mV}$, (b) $150 \mathrm{mV}$, and (c) $400 \mathrm{mV}$, and with the feedback protocol in the drive amplitude of (d) $0 \mathrm{mV}$, (e) $150 \mathrm{mV}$, and (f) $400 \mathrm{mV}$. Note that the axes scales are totally different between the two protocols.

$K_{i}^{P} \quad$ was only available in the postprocessing without any fast feedback processor. Thus, we used $K_{x}^{M}$ in the measurement-feedback control and $K_{x}^{P}$ in the analysis to, for instance, calculate the stochastic average of $K_{x}$. Figure 5 shows marginal probability density functions in the space spanned by $K_{x}^{P}$ and $K_{z}^{P}$ for both the random protocol and our measurement-feedback protocol. Apparently, the initial equilibrium state was isotropically distributed [see Figs. 5(a) and $5(\mathrm{~d})$ ]. On the other hand, the non-equilibrium steady states in the random protocol [see Figs. 5(b) and 5(c)] and our measurement-feedback protocol [see Figs. 5(e) and 5(f)] show biased distributions along $K_{x}>0$ directions due to the pseudorotation in parametric squeezing. We emphasize that the axes scales are totally different between the random protocol and our measurement-feedback protocol. Although the distribution is broadened in the random protocol because the parametric drive is blindly injected, the distribution in our protocol in the positive $K_{x}$ side is almost completely kept thanks to the switch-off operation.

\section{Net cooling effect and entropy production rates}

To unveil the net cooling effect hidden in our measurementfeedback experiment, we evaluate the entropy production rate from the heat bath, $\dot{\mathcal{S}}_{\text {bath }}$, and the entropy pumping rate $\dot{\mathcal{S}}_{\text {pump }}$ from the theoretical formulation in Eqs. (5) and (6). Note that the stochastic averages in Eqs. (5) and (6) are determined by using $K_{i}^{P} . \dot{\mathcal{S}}_{\text {bath }}$ is experimentally determined by

$$
\left\langle\dot{\mathcal{S}}_{\text {bath }}\right\rangle=\Gamma \frac{\left\langle K_{z}^{P}\right\rangle-\left\langle K_{z}^{P}\right\rangle_{0}}{\left\langle K_{z}^{P}\right\rangle_{0}},
$$

where $\langle\cdot\rangle_{0}$ denotes the stochastic average in the initial equilibrium state. In the same manner, we can obtain

$$
\left\langle\dot{\mathcal{S}}_{\text {pump }}\right\rangle=\sqrt{\frac{1}{2 \pi}} \frac{\Gamma \tilde{G}_{0}}{\sigma_{M}}\left\langle K_{z}^{P} \exp \left[-\frac{\left(K_{x}^{P}\right)^{2}}{2 \sigma_{M}^{2}}\right]\right\rangle .
$$

To estimate the entropy production rates, the effective drive strength $\tilde{G}_{0}$ and measurement noise deviation $\sigma_{M}$ are required. The $\tilde{G}_{0}$ was estimated from the noise reduction level in the random protocol shown in Fig. 4(b) (yellow circles). From the divergence condition $\tilde{G}_{0}=2$ in the random protocol, at which the noise reduction level changes from decreasing to increasing, we can determine $\tilde{G}_{0} / V_{\text {drive }}=10.4 \mathrm{~V}^{-1}$. The $\sigma_{M}$ was estimated to be $0.52 \pm 0.07$ from both $K_{x}^{M}$ and $K_{x}^{P}$ by assuming the Gaussian noise in measurement (see Appendix F).

The entropy production rates normalized by the mechanical damping rate $\Gamma$ are shown in Fig. 6(a). With increasing driving strength $\tilde{G}_{0}$, the entropy pumping rate $\dot{\mathcal{S}}_{\text {pump }}$ naturally increases with positive values. On the other hand, the entropy production rate in the thermal bath $\dot{\mathcal{S}}_{\text {bath }}$ decreases with negative values. This indicates that our measurement-feedback protocol successfully induces a net cooling effects where the system pulls the heat from the thermal bath to the feedback controller [see Fig. 6(b)]. Thus, this net cooling effect avoids heating due to the parametric driving, and results in stronger squeezing in its NESS because it allows us to inject stronger parametric driving over the limitation due to the divergence. Moreover, we can confirm the second-law-like inequality $\left\langle\dot{\mathcal{S}}_{\text {bath }}\right\rangle+\left\langle\dot{\mathcal{S}}_{\text {pump }}\right\rangle \geqslant 0$ from the experimental results. The total entropy production rate $\langle\dot{\Sigma}\rangle \equiv\left\langle\dot{\mathcal{S}}_{\text {bath }}\right\rangle+\left\langle\dot{\mathcal{S}}_{\text {pump }}\right\rangle$ apparently increases with increasing $\tilde{G}_{0}$. This is because the smaller $\tilde{G}_{0}$ purely induces the parametric squeezing with the net cooling [see Fig. 6(b)], while the larger $\tilde{G}_{0}$ intrinsically induces an additional heating in its pseudorotation despite successful operation of the protocol [see Fig. 6(c)].

It is intuitive that entropy production rates between our measurement-feedback protocol and the random protocol can be continuously related with respect to the measurement noise deviation $\sigma_{M}$ (i.e., $\sigma_{M} \rightarrow \infty$ corresponds to the random protocol). Thus, we numerically evaluate the entropy production rates $\left\langle\dot{\mathcal{S}}_{\text {bath }}\right\rangle$ and $\left\langle\dot{\mathcal{S}}_{\text {pump }}\right\rangle$ with different $\sigma_{M}=\left\{10^{-1}, 10^{0}, 10^{1}\right\}$ 
(a)

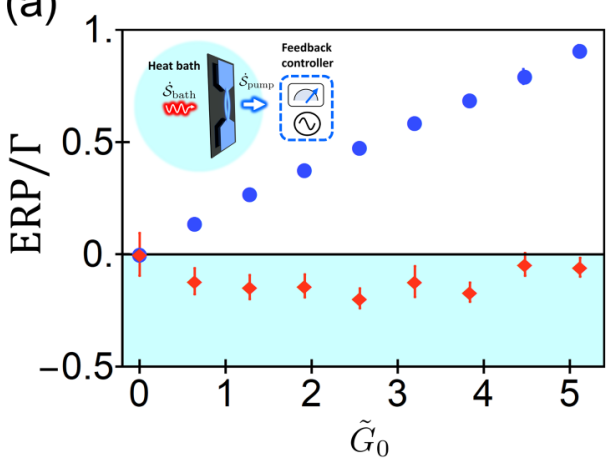

(b)

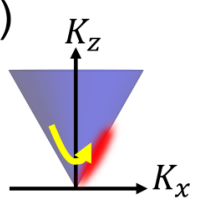

(c)

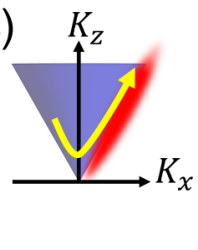

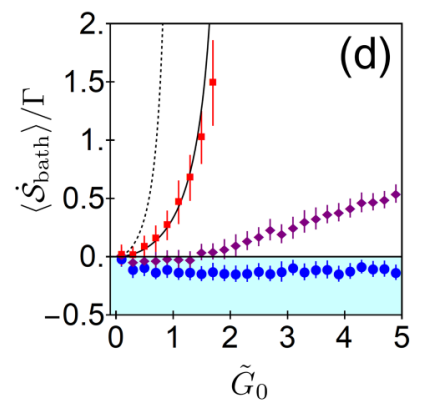

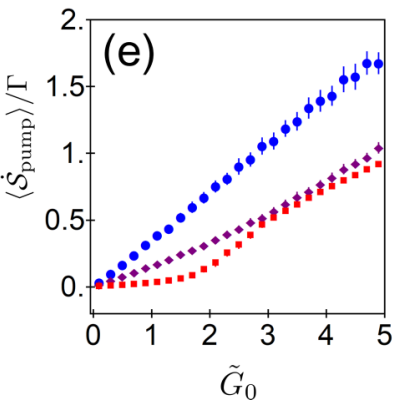

FIG. 6. (a) Entropy production rate (EPR) normalized by the mechanical damping rate $\Gamma$ in our measurement-feedback protocol. The red diamonds show the entropy production rates in the thermal bath $\left\langle\dot{\mathcal{S}}_{\text {bath }}\right\rangle / \Gamma$ and the blue dots show that in the feedback controller $\left\langle\dot{\mathcal{S}}_{\text {pump }}\right\rangle / \Gamma$ (i.e., entropy pumping rate). The blue shaded area corresponds to the cooling regime where the system operates as a cooler (conceptual image is shown in the inset). The error bars show the standard deviation in ten trials. [(b),(c)] Schematic of pseudorotation with (b) smaller $G_{0}$ and (c) larger $G_{0}$ in the Schwinger angularmomentum space. Numerically evaluated entropy production rates of (d) $\left\langle\dot{\mathcal{S}}_{\text {bath }}\right\rangle / \Gamma$ and (e) $\left\langle\dot{\mathcal{S}}_{\text {pump }}\right\rangle / \Gamma$. The dots, diamonds, and squares correspond to the noise deviation of $\sigma_{M}=10^{-1}, 10^{0}$, and $10^{1}$, respectively. The error bars show the standard deviation within fifty trials. The solid and dotted lines in (d) correspond to the entropy production rates via the continuous and random driving, respectively. The blue shaded area in (d) corresponds to the cooling regime.

[see Figs. 6(c) and 6(d)] and compare them with the analytical expression of $\left\langle\dot{\mathcal{S}}_{\text {bath }}\right\rangle$ in continuous and random driving (see Appendix G). As the measurement noise deviation increases, the slope of increment of $\left\langle\dot{\mathcal{S}}_{\text {bath }}\right\rangle$ become steep while the slope of increment of $\left\langle\dot{\mathcal{S}}_{\text {pump }}\right\rangle$ becomes gentle. This is because the measurement error induces the heating from parametric driving with the pseudorotation in $K_{x} \geqslant 0$, which decreases the influence of measurement and feedback (it becomes close to the random protocol). We emphasize again that our measurement-feedback scheme yields a noise-squeezed NESS in the cooling regime, whereas the conventional parametric scheme (continuous or random) yields it in the heating regime.

\section{DISCUSSION}

Our proof-of-principle experiment for the continuous measurement feedback control with Schwinger angular momentum was performed with additional white noise to improve the signal-to-noise ratio in the quadratic measurement in the
Doppler interferometer. The quadratic measurement can be extended to pure thermal fluctuation in mechanical resonators with Doppler interferometry by increasing the mechanical Q factor and decreasing effective mass in the mechanical modes [23]. Although the mechanical Q factor simply contributes to the signal-to-noise ratio as $\sqrt{Q}$ dependence, the inverse of effective mass linearly contributes to it. Thus, mechanical resonators with small effective mass (e.g., graphene drum resonators $[30,31])$ are suitable for performing our protocol with pure thermal motion with the Doppler interferometry. As an alternative approach, cavity optomechanical coupling in the unresolved sideband regime is also available for measuring higher-order harmonics in mechanical modes, and has been demonstrated for observing them in pure thermal motion [21,22]. Furthermore, extension to more higher-order variables and intermodal variables would open the way to more functionally control in the nonlinear measurement-feedback frameworks.

It is important to emphasize that experimental verification of the second-law-like inequality with the entropy pumping rate has been demonstrated only in linear measurementfeedback schemes [15]. In contrast, we investigated entropy production under fully quadratic measurement-feedback control of stochastic dynamics with a certain symmetry (i.e., certain geometry of variable space). Such a nonlinear nonequilibrium thermodynamic operation can be extended to the multimodal (e.g., two-mode mixing [32,33] and two-mode squeezing [34,35]) and the higher order (e.g., Duffing nonlinearity [36]) nonlinear operations by combining with an appropriate nonlinear measurement. Utilizing such rich intrinsic and external (measurement) nonlinearity in mechanical resonators might further promote the experimental verification of various types of thermodynamic limitations $[37,38]$ in nonlinear information thermodynamic machines.

\section{CONCLUSION}

In conclusion, we have demonstrated fully quadratic measurement-feedback control based on Schwinger angular momentum using a high-Q silicon nitride mechanical resonator by Doppler interferometry. A strong noise reduction level $-5.1 \pm 0.2 \mathrm{~dB}$ was achieved by suppressing the heating effect in parametric drive by well manipulating the probability distribution in Schwinger angular-momentum space. Furthermore, we have unveiled that our quadratic protocol enables us to perform the noise reduction in the cooling regime, where the parametric instability is completely removed, by investigating the entropy production rates. This nonlinear measurement-feedback control framework can be extended to investigating the general performance of various types of nonlinear information thermodynamic machine in the higher-order and multimodal nonlinear regime.

\section{ACKNOWLEDGMENT}

We thank Kensaku Chida for fruitful discussions. 


\section{APPENDIX A: DYNAMICS IN THE ROTATING FRAME WITH PARAMETRIC SQUEEZING}

The dynamics of displacement $X$ in a mechanical resonator with parametric force $F_{\mathrm{p}}$ is given by

$$
\ddot{X}+\Gamma \dot{X}+\left(\Omega^{2}+F_{\mathrm{p}}\right) X=F_{\mathrm{th}},
$$

where $\Gamma$ is the damping factor, $\Omega$ is the mechanical angular frequency, and the Langevin force $F_{\text {th }}$ is given by $\left\langle F_{\text {th }}(t) F_{\text {th }}\left(t^{\prime}\right)\right\rangle=2 k_{B} T \Gamma \delta\left(t-t^{\prime}\right)$. To induce the parametric squeezing, the parametric force has a double period of mechanical oscillation, i.e., $F_{\mathrm{p}}=-2 G_{0} \Omega \cos 2 \Omega t$ with the drive strength of $G_{0}$. The dynamics in rotating phase space spanned by linear quadratures $q$ and $p(X=q \cos \Omega t+p \sin \Omega t)$ is approximated by $\ddot{z} \ll \Omega \dot{z}(z=q, p)$ and $\Gamma / \Omega \ll 1$, that is the linear quadrature in a high-Q mode more slowly varies than the mechanical frequency as follows:

$$
\begin{aligned}
& -\Omega(2 \dot{q}+\Gamma q) \sin \Omega t+\Omega(2 \dot{p}+\Gamma p) \cos \Omega t \\
& -G_{0} \Omega[q(\cos \Omega t+\cos 3 \Omega t)+p(\sin 3 \Omega t-\sin \Omega t)]=F_{\text {th }} .
\end{aligned}
$$

To take into account the rotating term with $\Omega$, the Langevin force is split as $F_{\text {th }}=-f_{q} \sin \Omega t-f_{p} \cos \Omega t$ where $\left\langle f_{z}(t) f_{z}\left(t^{\prime}\right)\right\rangle=2 k_{B} T \Gamma \delta\left(t-t^{\prime}\right)$ is satisfied. This leads to the Langevin equations for each quadrature with the parametric squeezing as follows:

$$
\begin{aligned}
& \dot{q}=-\frac{\Gamma}{2} q+\frac{G_{0}}{2} p+\sqrt{\tilde{\beta} \Gamma} \xi_{q}, \\
& \dot{p}=-\frac{\Gamma}{2} p+\frac{G_{0}}{2} q+\sqrt{\tilde{\beta} \Gamma} \xi_{p},
\end{aligned}
$$

where $\tilde{\beta} \equiv k_{B} T / 2 \Omega^{2}$. Note that the effective rotating-framed Hamiltonian is given by $\mathcal{H}_{\text {eff }}=G_{0}\left(p^{2}-q^{2}\right) / 4$.

\section{APPENDIX B: HEATING EFFECT IN PARAMETRIC SQUEEZING}

Heating in continuous parametric squeezing can be simply seen in the change in the Shannon entropy between the initial and final equilibrium states, which is given by

$$
\Delta \mathcal{H}_{\mathrm{S}}=\frac{1}{2} \ln \frac{\left|\Sigma_{f}\right|}{\left|\Sigma_{i}\right|},
$$

where $\left|\Sigma_{i}\right|$ and $\left|\Sigma_{f}\right|$ are determinants of covariant matrices in the initial equilibrium state and final squeezed state, respectively. From Langevin equations for continuous parametric squeezing Eqs. (A3) and (A4), the determinant of the covariant matrix in the final squeezed state is given by

$$
\left|\Sigma_{f}\right|=\frac{16 \tilde{\beta} \Gamma}{\left(\Gamma^{2}-G_{0}^{2}\right)^{2}} .
$$

This leads to

$$
\Delta \mathcal{H}_{\mathrm{S}}=\ln \frac{1}{1-G_{0}^{2} / \Gamma^{2}} .
$$

Since the Shannon entropy monotonically increases in the stable squeezing regime $G_{0}<\Gamma$, the system (i.e., mechanical resonator) is totally heated up due to the parametric squeezing.

\section{APPENDIX C: ENTROPY PRODUCTION IN OUR PROTOCOL BY MEANS OF PATH INTEGRAL}

Total entropy production $\Sigma$, which is always non-negative, is defined by the Kullback-Leibler divergence between the forward probability distribution and the inverse probability distribution as

$$
\Sigma=\ln \frac{\mathcal{P}_{\text {fwd }}}{\mathcal{P}_{\text {inv }}} \geqslant 0 .
$$

In the case of the continuous measurement-feedback control, entropy production has been investigated in a coarse-grained dynamics, where the memory degree of freedom in measurement is coarse-grained in its equation of motion [13,14,25]. The inverse probability in the coarse-grained dynamics was defined as a probability with "conjugate" dynamics, in which the time-reversal parity of feedback cooling forces is defined to be positive $[13,25]$. From the path-integral formalism, the entropy production in the non-equilibrium steady state (i.e., change in the Shannon entropy is zero) is expressed by

$$
\Sigma=\int d s \dot{\mathcal{S}}_{\text {bath }}(s)+\int d s \dot{\mathcal{S}}_{\text {pump }}(s),
$$

where $\dot{\mathcal{S}}_{\text {bath }}$ and $\dot{\mathcal{S}}_{\text {pump }}$ are the entropy production rates in thermal bath and controller. The latter has been referred to as "entropy pumping" [13,25], which gives second-law like inequality including the influence of information extraction as

$$
\dot{\mathcal{S}}_{\text {bath }} \geqslant-\dot{\mathcal{S}}_{\text {pump }} \text {. }
$$

To derive the actual expression of entropy production in our quadratic measurement feedback, we start from the Langevin equation in the laboratory frame with the displacement $X$, the momentum $P$, and the state of feedback memory $M$ as follows:

$$
\begin{gathered}
\dot{X}=P, \\
\dot{P}+A(X, P, M, t)=\mathcal{F}_{\text {th }} .
\end{gathered}
$$

The term of the equation of motion $A(X, P, t)$ is given by

$$
A(X, P, M, t)=\Gamma P+\Omega^{2} X-2 G_{0} \Omega f(M) \cos 2 \Omega t X,
$$

where $\Gamma$ is the damping rate, and $\Omega$ is the angular frequency of the mechanical resonator. Note that we set the effective mass to be unity in the following discussion for simplicity (replacing $k_{B}$ by $k_{B} / m_{\text {eff }}$ provides us the exact expressions with the effective mass of mechanical resonator $\left.m_{\mathrm{eff}}\right)$. The Langevin force $\mathcal{F}_{\text {th }}$ satisfies $\left\langle\mathcal{F}_{\text {th }}\left(t^{\prime}\right) \mathcal{F}_{\text {th }}(t)\right\rangle=2 k_{B} T \Gamma \delta\left(t-t^{\prime}\right)$ with the Boltzmann constant $k_{B}$ and the temperature of the environment $T$. The protocol of measurement and feedback is expressed by the feedback function $f(M)$ with the memory value $M$. In our protocol, the feedback function is given by a Heaviside function $\theta(\cdot)$ as $f(M)=\theta(M)$. Analytical difficulty in the discontinuity of the Heaviside function is avoided by taking into account the finite measurement noise, which is assumed as Gaussian white noise. Thus, the conditional probability without feedback delay,

$$
\mathcal{P}(M \mid \mathcal{M}(X, P))=\frac{1}{\sqrt{2 \pi \sigma_{M}^{2}}} \exp \left[-\frac{(\mathcal{M}(X, P)-M)^{2}}{2 \sigma_{M}^{2}}\right]
$$


is suitable for modeling our measurement-feedback loop, where $\mathcal{M}(X, P)$ is the target variable in the measurement, and $\sigma_{M}$ is the standard deviation of the Gaussian noise. By multiplying Eq. (C7) with Eq. (C5) and integrating both sides with respect to $M$, a coarse-grained dynamics is represented as follows:

$$
\dot{P}+A(X, P, t)=F_{\text {th }},
$$

$$
\begin{aligned}
& A(X, P, t) \\
& \quad=\Gamma P+\Omega^{2} X-G_{0} \Omega \operatorname{erfc}\left(\frac{\mathcal{M}(X, P)}{\sqrt{2} \sigma_{M}}\right) \cos 2 \Omega t X .
\end{aligned}
$$

where $\operatorname{erfc}(\cdot)$ is a complementary error function reflecting the switching operation with finite measurement noise.

From the Fokker-Planck equation,

$$
\partial_{t} \mathcal{P}=\mathcal{L} \mathcal{P}
$$

with an operator

$$
\mathcal{L} \equiv \partial_{P} A(X, P, t)+k_{B} T \partial_{P}^{2}-\partial_{X}(P / m),
$$

we achieve the forward transition probability in stochastic path from the initial condition $\left(X_{0}, P_{0}, t_{0}\right)$ as

$$
\begin{aligned}
\mathcal{P}\left(X, P, t \mid X_{0}, P_{0}, t_{0}\right) \\
=\mathcal{B} \exp \left[-\frac{1}{4 k_{B} T \Gamma} \int_{t_{0}}^{t} d s(\dot{P}(s)+A(X(s), P(s), s))^{2}\right] \\
\quad \times \exp \left[\frac{1}{2} \int_{t_{0}}^{t} d s \frac{\partial A(X, P, t)}{\partial P}\right],
\end{aligned}
$$

where $\mathcal{B}$ is a constant. The first exponential term corresponds to the Onsager-Machlup function, and the second term is derived from the Ito formula [26]. Thus, the conjugate dynamics is given by

$$
\begin{aligned}
& \mathcal{P}^{*}\left(X_{0}, P_{0}, t \mid X, P, t_{0}\right) \\
& =\mathcal{B} \exp \left[-\frac{1}{4 k_{B} T \Gamma} \int_{t_{0}}^{t} d s\left(\dot{P}(s)+A^{*}(X(s), P(s), s)\right)^{2}\right] \\
& \quad \times \exp \left[\frac{1}{2} \int_{t_{0}}^{t} d s\left(\frac{\partial A(X, P, t)}{\partial P}\right)^{*}\right]
\end{aligned}
$$

where ${ }^{*}$ denotes a time-reversal operation. Because the entropy production is given by the ratio between Eqs. (C12) and (C13), the time-reversal parity of $A(X, P, t)$ is crucial. From Eq. (C8), $A(X, P, t)$ is given by

$$
\begin{aligned}
A(X, P, t)= & \Gamma P+\Omega^{2} X-G_{0} \Omega \operatorname{erfc}\left(\frac{\mathcal{M}(X, P)}{\sqrt{2} \sigma_{M}}\right) \cos 2 \Omega t X \\
= & \Gamma P+\Omega^{2} X-G_{0} \Omega\left[1-\operatorname{erf}\left(\frac{\mathcal{M}(X, P)}{\sqrt{2} \sigma_{M}}\right)\right] \\
& \times \cos 2 \Omega t X,
\end{aligned}
$$

where the complementary error function is decomposed to a constant and an odd function (error function). This decomposition is crucial for calculating its conjugate dynamics as

$$
\begin{aligned}
A^{*}(X, P, t)= & \Gamma P^{*}+\Omega^{2} X^{*} \\
& -G_{0} \Omega\left[1-t_{\mathrm{P}} \operatorname{erf}\left(\frac{\mathcal{M}^{*}(X, P)}{\sqrt{2} \sigma_{M}}\right)\right] \cos 2 \Omega t X^{*}, \\
= & -\Gamma P+\Omega^{2} X \\
& -G_{0} \Omega\left[1-\operatorname{erf}\left(\frac{\mathcal{M}(X, P)}{\sqrt{2} \sigma_{M}}\right)\right] \cos 2 \Omega t X .
\end{aligned}
$$

Here, $t_{p}=\{-1,1\}$ is determined by the time-reversal parity of the target variable $\mathcal{M}(X, P)$, where $t_{p}=1(-1)$ when the $\mathcal{M}^{*}(X, P)=\mathcal{M}(X, P)\left[\mathcal{M}^{*}(X, P)=-\mathcal{M}(X, P)\right]$. Thus, regardless of the time-reversal parity of the target variable, the feedback force is treated as a reversible force in the conjugate dynamics [13]. By using this probability in the conjugate dynamics,

$$
\begin{aligned}
\exp [\Sigma]= & \frac{\mathcal{P}\left(X, P, t \mid X_{0}, P_{0}, t_{0}\right)}{\mathcal{P}^{*}\left(X_{0}, P_{0}, t \mid X, P, t_{0}\right)} \\
= & \exp \left[-\frac{1}{k_{B} T} \int d s P \circ\left(\dot{P}+\Omega^{2} X\right.\right. \\
& \left.\left.-G_{0} \Omega\left[1-\operatorname{erf}\left(\frac{\mathcal{M}(X, P)}{\sqrt{2} \sigma_{M}}\right)\right] \cos 2 \Omega t X\right)\right] \\
& \times \exp \left[\frac{2 G_{0} \Omega}{\sqrt{2 \pi} \sigma_{M}} \int d s \exp \left[-\left(\frac{\mathcal{M}(X, P)}{\sqrt{2} \sigma_{M}}\right)^{2}\right]\right. \\
& \left.\times \frac{\partial \mathcal{M}(X, P)}{\partial P} \circ X \cos 2 \Omega t\right],
\end{aligned}
$$

where $\circ$ explicitly denotes Stratonovich integral. The first exponential term corresponds to the entropy production in the thermal bath, and the second exponential term corresponds to the entropy production in the controller. The entropy production rate, which is directly achieved by taking the time derivative in Eq. (C16) can be expressed as follows:

$$
\begin{gathered}
\dot{\Sigma}=\dot{\mathcal{S}}_{\text {bath }}+\dot{\mathcal{S}}_{\text {pump }}, \\
\dot{\mathcal{S}}_{\text {bath }}=-\frac{1}{k_{B} T} P \circ\left(-\Gamma P+F_{\text {th }}\right), \\
\dot{\mathcal{S}}_{\text {pump }}=-\frac{2 G_{0} \Omega}{\sqrt{2 \pi} \sigma_{M}} \exp \left[-\left(\frac{\mathcal{M}(X, P)}{\sqrt{2} \sigma_{M}}\right)^{2}\right] \\
\times \frac{\partial \mathcal{M}(X, P)}{\partial P} \circ X \cos 2 \Omega t .
\end{gathered}
$$

Because the target variable corresponds to $K_{x}$, which is given in the rotating frame, we perform the rotating wave approximation to linearize the transformation from the laboratory frame $(X, P)$ to the rotating frame $(q, p)$ as

$$
\begin{gathered}
X=q \cos \Omega t+p \sin \Omega t, \\
P / \Omega \approx-q \sin \Omega t+p \cos \Omega t .
\end{gathered}
$$


Thus, the momentum derivative of the target variable in Eq. (C19) is evaluated by

$$
\begin{aligned}
\frac{\partial \mathcal{M}(X, P)}{\partial P} & \approx \frac{\partial q}{\partial P} \frac{\partial \mathcal{M}(q, p)}{\partial q}+\frac{\partial p}{\partial P} \frac{\partial \mathcal{M}(q, p)}{\partial p} \\
& =\frac{1}{2 \Omega}(q \cos \Omega t-p \sin \Omega t) .
\end{aligned}
$$

By using the following approximation,

$$
P^{2} \approx 2 \Omega^{2} K_{z},
$$

$$
(q \cos \Omega t-p \sin \Omega t) X \cos 2 \Omega t /(2 \Omega) \approx K_{z} / 2,
$$

we obtain the expressions of stochastic average of entropy production,

$$
\begin{array}{r}
\left\langle\dot{\mathcal{S}}_{\text {bath }}\right\rangle \approx \frac{2 \Omega^{2} \Gamma}{k_{B} T}\left(\left\langle K_{z}\right\rangle-\frac{k_{B} T}{2 \Omega^{2}}\right), \\
\left\langle\dot{\mathcal{S}}_{\text {pump }}\right\rangle \approx \sqrt{\frac{1}{2 \pi}} \frac{\tilde{G}_{0} \Gamma}{\sigma_{M}}\left\langle K_{z} \exp \left(-\frac{K_{x}^{2}}{2 \sigma_{M}^{2}}\right)\right\rangle .
\end{array}
$$

\section{APPENDIX D: ENTROPY PRODUCTION IN OUR PROTOCOL FROM PROBABILITY CURRENTS}

Although the path-integral formalism shown in Appendix C provides us a complete expression of entropy production with exact physical meaning, attempting to calculate it via the another simple formalism directly from the coarse-grained Fokker-Planck equation via probability currents $[14,27,28]$ is worthwhile to confirm our formula in Eqs. (C25) and (C26). The Fokker-Planck equation is re-expressed by probability currents $J_{z}(z=X, P)$ as

$$
\begin{gathered}
\partial_{t} \mathcal{P}=-\sum_{z} \partial_{z} J_{z}, \\
J_{X}=-P \mathcal{P}, \\
J_{P}=-\Gamma P \mathcal{P}-\Omega^{2} X \mathcal{P} \\
+G_{0} \Omega\left[1-\operatorname{erf}\left(\frac{\mathcal{M}(X, P)}{\sqrt{2} \sigma_{M}}\right)\right] \cos 2 \Omega t X \mathcal{P} \\
-k_{B} T \Gamma \partial_{P} \mathcal{P} .
\end{gathered}
$$

Here, we split the momentum current $J_{P}$ into two in terms of the time-reversal parity, the same as discussed in Appendix C, in which the feedback force is regarded as a reversible force,

$$
\begin{gathered}
J_{P}^{\mathrm{rev}}=-\Omega^{2} X \mathcal{P}, \\
+G_{0} \Omega\left[1-\operatorname{erf}\left(\frac{\mathcal{M}(X, P)}{\sqrt{2} \sigma_{M}}\right) \cos 2 \Omega t X\right] \mathcal{P}, \\
J_{P}^{\mathrm{irr}}=\left[-\Gamma P-k_{B} T \Gamma \partial_{P}\right] \mathcal{P} .
\end{gathered}
$$

Introducing Shannon entropy $\langle\mathcal{S}\rangle \equiv-\int d X d P \mathcal{P} \ln \mathcal{P}$ using a relationship $\partial_{t}\langle\mathcal{S}\rangle=-\int d X d P\left(\partial_{t} \mathcal{P}\right) \ln \mathcal{P}=$ $-\int d X d P\left(\partial_{t} \mathcal{P}\right)(1+\ln \mathcal{P})$,

$$
\begin{aligned}
\frac{\partial\langle\mathcal{S}\rangle}{\partial t} & =\int d X d P\left(\sum_{z} \partial_{z} J_{z}\right)(\ln \mathcal{P}+1) \\
& =-\int d X d P \sum_{z} \frac{J_{z} \partial_{z} \mathcal{P}}{\mathcal{P}} .
\end{aligned}
$$

Here, the second equation is derived by using a partial integral and removing the boundary integral because the probability density function on the boundary is assumed to take zero. Note that

$$
\int d X d P \frac{J_{x} \partial_{X} \mathcal{P}}{\mathcal{P}}=-\int d X d P \mathcal{P} \partial X(-P)=0 .
$$

Moreover, by using the relationship $\partial_{P} \mathcal{P}=-\frac{1}{k_{B} T \Gamma}\left(J_{P}^{\mathrm{irr}}+\Gamma P\right)$ from Eq. (D5), it reduces to

$$
\begin{aligned}
\frac{\partial\langle\mathcal{S}\rangle}{\partial t}= & \frac{1}{k_{B} T \Gamma} \int d X d P \frac{1}{\mathcal{P}}\left[\left(J_{P}^{\mathrm{irr}}\right)^{2}+\Gamma P J_{P}^{\mathrm{irr}} \mathcal{P}\right] \\
& -\int d X d P \frac{1}{\mathcal{P}} J_{P}^{\mathrm{rev}} \partial_{P} \mathcal{P} \\
= & \left\langle\dot{\mathcal{S}}_{\text {tot }}\right\rangle-\left\langle\dot{\mathcal{S}}_{\text {bath }}\right\rangle-\left\langle\dot{\mathcal{S}}_{\text {pump }}\right\rangle .
\end{aligned}
$$

The first term in Eq. (D8) corresponds to the non-negative entropy production,

$$
\left\langle\dot{\mathcal{S}}_{\mathrm{tot}}\right\rangle \equiv \frac{1}{k_{B} T \Gamma} \int d X d P \frac{\left(J_{P}^{\mathrm{irr}}\right)^{2}}{\mathcal{P}} \geqslant 0,
$$

which obviously posses the second-law-like inequality,

$$
\frac{\partial\langle\mathcal{S}\rangle}{\partial t}+\left\langle\dot{\mathcal{S}}_{\text {bath }}\right\rangle+\left\langle\dot{\mathcal{S}}_{\text {pump }}\right\rangle \geqslant 0 .
$$

The second term in Eq. (D8) corresponds to the entropy production rate due to the existence of irreversible currents. It can be expanded to

$$
\begin{aligned}
\left\langle\dot{\mathcal{S}}_{\text {bath }}\right\rangle & \equiv \frac{1}{k_{B} T \Gamma} \int d X d P \Gamma P \circ\left(\Gamma P+k_{B} T \Gamma \partial_{P}\right) \mathcal{P} \\
& =\frac{1}{k_{B} T \Gamma}\left(\Gamma^{2}\left\langle P^{2}\right\rangle-k_{B} T \Gamma^{2}\right) \\
& \approx \frac{2 \Omega^{2} \Gamma}{k_{B} T}\left(\left\langle K_{z}\right\rangle-\frac{k_{B} T}{2 \Omega^{2}}\right)
\end{aligned}
$$

where $\tilde{G}_{0} \equiv G_{0} / \Gamma$ is notated. The approximation in Eq. (D11) is equivalent to that in Eqs. (C25) and (C26). Apparently, we can confirm that Eq. (D11) completely corresponds to the entropy production in the thermal bath, Eq. (C25), derived in the path-integral formalism.

The third term in Eq. (D8) is regarded as the entropy production rate thanks to the presence of measurement and 

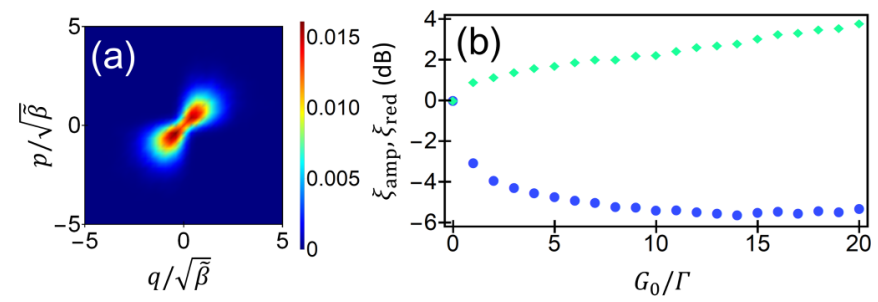

FIG. 7. (a) Numerically calculated probability density function under error free protocol with $\sigma_{M}=10^{-10}$ and $G_{0} / \Gamma=14$ where the color map corresponds to the probability density. The non-Gaussian distribution, which is similar to the experimental result [see Fig. 4(c)] is achieved. (b) Numerically calculated noise reduction (blue dots) and amplification (green diamond) level with respect to $G_{0} / \Gamma$. The noise reduction level is converged to a lower bound about $-6 \mathrm{~dB}$ with increasing the parametric gain.

feedback, i.e., the entropy pumping rate, simplified as

$$
\begin{aligned}
\left\langle\dot{\mathcal{S}}_{\text {pump }}\right\rangle & =\int d X d P \frac{1}{\mathcal{P}} J_{P}^{\mathrm{rev}} \partial_{P} \mathcal{P}, \\
& =G_{0} \Omega \cos 2 \Omega t\left\langle\partial_{P}\left[\operatorname{erf}\left(\frac{\mathcal{M}(X, P)}{\sqrt{2} \sigma_{M}}\right) \circ X\right]\right\rangle, \\
& \approx \sqrt{\frac{1}{2 \pi}} \frac{\tilde{G}_{0} \Gamma}{\sigma_{M}}\left\langle K_{z} \exp \left(-\frac{K_{x}^{2}}{2 \sigma_{M}^{2}}\right)\right\rangle .
\end{aligned}
$$

This expression is also equivalent to that in Eq. (C26) derived in the path-integral formalism.

\section{APPENDIX E: NUMERICAL RESULTS IN THE NON-EQUILIBRIUM STEADY STATE}

To clarify the steady-state distribution in the ideal case, we numerically solve the coarse-grained Langevin equation in the rotating frame:

$$
\begin{aligned}
& \dot{q}=-\frac{\Gamma}{2} q+\frac{G_{0}}{4} \operatorname{erfc}\left(\frac{K_{x}}{\sqrt{2} \sigma_{M}}\right) p+\sqrt{\tilde{\beta} \Gamma} \xi_{q} \\
& \dot{p}=-\frac{\Gamma}{2} p+\frac{G_{0}}{4} \operatorname{erfc}\left(\frac{K_{x}}{\sqrt{2} \sigma_{M}}\right) q+\sqrt{\tilde{\beta} \Gamma} \xi_{p}
\end{aligned}
$$

where $\sigma_{M}=10^{-10}$ to discuss the error free case (the notation is the same defined in Appendix A). Figure 7 shows the probability density function of the NESS with $G_{0} / \Gamma=14$ where the noise reduction level of $-5.5 \pm 0.2 \mathrm{~dB}$ is achieved over the $-3-\mathrm{dB}$ limit. Apparently, the probability density function retains the non-Gaussian distribution as well as that experimentally obtained. With increasing $G_{0}$, the noise reduction level is converged around $-6 \mathrm{~dB}$, the noise amplification level gradually increases. This shows good agreements achieved in the experiment [see Fig. 4(d)].

Although we confirm that our theoretical model with no feedback delay shows good agreement to the experimental results, here we briefly discuss the contribution of the feedback delay. There exists two contributions: One is the measurement error due to the phase shift, and another one is the non-Markovian effect. The former, measurement error due to the phase shift, occurs because the measurement quantity is changed to $K_{x} \cos 2 \Omega \tau+K_{y} \sin 2 \Omega \tau$ with the finite delay $\tau$ from the ideal case of $K_{x}$. Because of the orthogonal- ity between $K_{x}$ and $K_{y}$, the term of $K_{y} \sin 2 \Omega \tau$ makes the measurement error for the pseudorotation around $K_{y}$ in our protocol. For instance, the large delay with $2 \Omega \tau=\pi / 2$ brings the operation in random where the noise reduction level is limited at $-3 \mathrm{~dB}$. The latter, non-Markovian effect, could appear in the further large delay (e.g., $2 \Omega \tau=2 \pi$ ), which induces the breakdown of the feedback cooling in linear measurement and feedback [15].

\section{APPENDIX F: ESTIMATION OF $\sigma_{M}$}

To estimate a noise deviation in the measurement $\sigma_{M}$, an observation is modelled by

$$
K_{x}^{M}=a\left(K_{x}+\sigma_{M} \xi_{M}\right),
$$

where $a$ shows an arbitrary coefficient in measurement, and $\xi$ shows a Markovian noise with $\left\langle\xi(t) \xi\left(t^{\prime}\right)\right\rangle=\delta\left(t-t^{\prime}\right)$. Because the true value of $K_{x}$ can be approximated by $K_{x}^{P}$, which is the postprocessed value, $\sigma_{M}$ can be determined by

$$
\begin{gathered}
\sigma_{M}=\sqrt{\frac{\left\langle\left(K_{x}^{M}\right)^{2}\right\rangle}{a^{2}}-\left\langle\left(K_{x}^{P}\right)^{2}\right\rangle} \\
a=\frac{\left\langle K_{x}^{M} K_{x}^{P}\right\rangle}{\left\langle\left(K_{x}^{P}\right)^{2}\right\rangle}
\end{gathered}
$$

from the experimental data without any driving (i.e., $\left\langle K_{x}^{M}\right\rangle=$ $\left.\left\langle K_{x}^{P}\right\rangle=0\right)$. As a result, $\sigma_{M}$ is determined to be $0.52 \pm 0.07$.

\section{APPENDIX G: ENTROPY PRODUCTION IN CONTINUOUS DRIVING AND RANDOM DRIVING}

In the case of continuous driving [i.e., the feedback function becomes unity, $f(m)=1$ ], the expression of entropy production is straightforwardly derived because it only contains the contribution of the entropy production in the thermal bath, $\dot{\mathcal{S}}_{\text {bath }}^{\mathrm{C}}$. Thus, $\left\langle\dot{\mathcal{S}}_{\text {bath }}^{\mathrm{C}}\right\rangle$ is achieved with the same definition given in Eq. (C25). In the same manner, the entropy production with the random protocol, in which the feedback function is given by $f(m)=\xi_{R}$ with the random integer $\xi_{R} \equiv\{0,1\}$ can be formulated by taking into account the contribution from the thermal bath $\left\langle\dot{\mathcal{S}}_{\text {bath }}^{\mathrm{R}}\right\rangle$. Because entropy production is defined as the ratio between the forward and backward probability, we consider the minimum entropy production as that under the random switching. In other words, the force by the random switching is regarded as reversible in its conjugate dynamics, and as a result the total entropy production just consists of the entropy production in thermal bath $[13,25]$. Consequently, this means $\left\langle\dot{\mathcal{S}}_{\text {bath }}^{\mathrm{R}}\right\rangle$ can be calculated from Eq. (C25). $\left\langle\dot{\mathcal{S}}_{\text {bath }}^{\mathrm{C}}\right\rangle$ and $\left\langle\dot{\mathcal{S}}_{\text {bath }}^{\mathrm{R}}\right\rangle$ can be analytically calculated by solving the following Langevin equation in the rotating frame:

$$
\begin{gathered}
\left\langle\dot{K}_{x}\right\rangle=-\Gamma\left\langle K_{x}\right\rangle+\alpha G_{0}\left\langle K_{z}\right\rangle, \\
\left\langle\dot{K}_{z}\right\rangle=-\Gamma\left\langle K_{z}\right\rangle+\alpha G_{0}\left\langle K_{x}\right\rangle+\Gamma K_{0},
\end{gathered}
$$

where $K_{0} \equiv k_{B} T / 2 \Omega^{2}$, and $\alpha$ is a factor defined by $\alpha=1$ or $\alpha=1 / 2$ in case of continuous driving or random driving, 
respectively. The steady-state solutions are given by

$$
\left\langle K_{z}\right\rangle=\frac{K_{0}}{1-\alpha^{2} \tilde{G}_{0}^{2}}, \quad\left\langle K_{x}\right\rangle=\frac{\alpha \tilde{G}_{0} K_{0}}{1-\alpha^{2} \tilde{G}_{0}^{2}} .
$$

By substituting them into Eq. (C25), the entropy production rates under the random protocol are analytically expressed as follows:

$$
\begin{aligned}
\left\langle\dot{\mathcal{S}}_{\text {bath }}^{\mathrm{C}}\right\rangle & =\frac{\Gamma \tilde{G}_{0}^{2}}{1-\tilde{G}_{0}^{2},} \\
\left\langle\dot{\mathcal{S}}_{\text {bath }}^{\mathrm{R}}\right\rangle & =\frac{\Gamma \tilde{G}_{0}^{2} / 4}{1-\tilde{G}_{0}^{2} / 4}
\end{aligned}
$$

It is obvious that the entropy production in continuous driving (random driving) holds a divergence at $\tilde{G}_{0}=1\left(\tilde{G}_{0}=2\right)$. This divergence occurs because the thermal bath cannot absorb the heat from the parametric driving due to $G_{0} \geqslant \Gamma$ (or $\left.\tilde{G}_{0} / 2 \geqslant \Gamma\right)$.

[1] J. M. Parrondo, J. M. Horowitz, and T. Sagawa, Thermodynamics of information, Nat. Phys. 11, 131 (2015).

[2] S. Ciliberto, Experiments in Stochastic Thermodynamics: Short History and Perspectives, Phys. Rev. X 7, 021051 (2017).

[3] D. Andrieux, P. Gaspard, S. Ciliberto, N. Garnier, S. Joubaud, and A. Petrosyan, Entropy Production and Time Asymmetry in Nonequilibrium Fluctuations, Phys. Rev. Lett. 98, 150601 (2007).

[4] S. Toyabe, T. Sagawa, M. Ueda, E. Muneyuki, and M. Sano, Experimental demonstration of information-to-energy conversion and validation of the generalized Jarzynski equality, Nat. Phys. 6, 988 (2010).

[5] J. Gieseler, R. Quidant, C. Dellago, and L. Novotny, Dynamic relaxation of a levitated nanoparticle from a non-equilibrium steady state, Nat. Nanotechnol. 9, 358 (2014).

[6] F. Douarche, S. Ciliberto, A. Petrosyan, and I. Rabbiosi, An experimental test of the Jarzynski equality in a mechanical experiment, Europhys. Lett. 70, 593 (2005).

[7] M. Bonaldi, L. Conti, P. De Gregorio, L. Rondoni, G. Vedovato, A. Vinante, M. Bignotto, M. Cerdonio, P. Falferi, N. Liguori, S. Longo, R. Mezzena, A. Ortolan, G. A. Prodi, F. Salemi, L. Taffarello, S. Vitale, and J.-P. Zendri, Nonequilibrium SteadyState Fluctuations in Actively Cooled Resonators, Phys. Rev. Lett. 103, 010601 (2009).

[8] M. Serra-Garcia, A. Foehr, M. Molerón, J. Lydon, C. Chong, and C. Daraio, Mechanical Autonomous Stochastic Heat Engine, Phys. Rev. Lett. 117, 010602 (2016).

[9] M. Rossi, L. Mancino, G. T. Landi, M. Paternostro, A. Schliesser, and A. Belenchia, Experimental Assessment of Entropy Production in a Continuously Measured Mechanical Resonator, Phys. Rev. Lett. 125, 080601 (2020).

[10] P.-F. Cohadon, A. Heidmann, and M. Pinard, Cooling of a Mirror by Radiation Pressure, Phys. Rev. Lett. 83, 3174 (1999).

[11] D. Kleckner and D. Bouwmeester, Sub-Kelvin optical cooling of a micromechanical resonator, Nature (London) 444, 75 (2006).

[12] M. Poggio, C. L. Degen, H. J. Mamin, and D. Rugar, Feedback Cooling of a Cantilever's Fundamental Mode Below $5 \mathrm{mK}$, Phys. Rev. Lett. 99, 017201 (2007).

[13] T. Munakata and M. L. Rosinberg, Entropy Production and Fluctuation Theorems for Langevin Processes Under
Continuous Non-Markovian Feedback Control, Phys. Rev. Lett. 112, 180601 (2014).

[14] J. M. Horowitz and H. Sandberg, Second-law-like inequalities with information and their interpretations, New J. Phys. 16, 125007 (2014).

[15] M. Debiossac, D. Grass, J. J. Alonso, E. Lutz, and N. Kiesel, Thermodynamics of continuous non-Markovian feedback control, Nat. Commun. 11, 1360 (2020).

[16] A. Vinante and P. Falferi, Feedback-Enhanced Parametric Squeezing of Mechanical Motion, Phys. Rev. Lett. 111, 207203 (2013).

[17] M. Poot, K. Y. Fong, and H. X. Tang, Classical non-Gaussian state preparation through squeezing in an optoelectromechanical resonator, Phys. Rev. A 90, 063809 (2014).

[18] D. Rugar and P. Grütter, Mechanical Parametric Amplification and Thermomechanical Noise Squeezing, Phys. Rev. Lett. 67, 699 (1991).

[19] I. Mahboob, H. Okamoto, and H. Yamaguchi, An electromechanical Ising Hamiltonian, Sci. Adv. 2, e1600236 (2016).

[20] M. J. Seitner, M. Abdi, A. Ridolfo, M. J. Hartmann, and E. M. Weig, Parametric Oscillation, Frequency Mixing, and Injection Locking of Strongly Coupled Nanomechanical Resonator Modes, Phys. Rev. Lett. 118, 254301 (2017).

[21] G. Brawley, M. Vanner, P. E. Larsen, S. Schmid, A. Boisen, and W. Bowen, Nonlinear optomechanical measurement of mechanical motion, Nat. Commun. 7, 10988 (2016).

[22] R. Leijssen, G. R. La Gala, L. Freisem, J. T. Muhonen, and E. Verhagen, Nonlinear cavity optomechanics with nanomechanical thermal fluctuations, Nat. Commun. 8, 16024 (2017).

[23] M. Asano, R. Ohta, T. Aihara, T. Tsuchizawa, H. Okamoto, and H. Yamguchi, Optically probing Schwinger angular momenta in a micromechanical resonator, Phys. Rev. A 100, 053801 (2019).

[24] J. Schwinger, On Angular Momentum, in Quantum Theory of Angular Momentum, edited by L. C. Biedenharm and H. van Dam (Academic Press, New York, 1965).

[25] T. Munakata and M. Rosinberg, Entropy production and fluctuation theorems under feedback control: The molecular refrigerator model revisited, J. Stat. Mech.: Theory Exp. (2012) P05010.

[26] A. Imparato and L. Peliti, Fluctuation relations for a driven Brownian particle, Phys. Rev. E 74, 026106 (2006). 
[27] T. Tomé and M. J. de Oliveira, Entropy production in irreversible systems described by a Fokker-Planck equation, Phys. Rev. E 82, 021120 (2010).

[28] R. E. Spinney and I. J. Ford, Entropy production in full phase space for continuous stochastic dynamics, Phys. Rev. E 85, 051113 (2012).

[29] H. Nha and J. Kim, Entanglement criteria via the uncertainty relations in su(2) and su(1,1) algebras: Detection of non-Gaussian entangled states, Phys. Rev. A 74, 012317 (2006).

[30] J. S. Bunch, A. M. Van Der Zande, S. S. Verbridge, I. W. Frank, D. M. Tanenbaum, J. M. Parpia, H. G. Craighead, and P. L. McEuen, Electromechanical resonators from graphene sheets, Science 315, 490 (2007).

[31] M. Will, M. Hamer, M. Muller, A. Noury, P. Weber, A. Bachtold, R. Gorbachev, C. Stampfer, and J. Guttinger, High quality factor graphene-based two-dimensional heterostructure mechanical resonator, Nano Lett. 17, 5950 (2017).

[32] H. Okamoto, A. Gourgout, C.-Y. Chang, K. Onomitsu, I. Mahboob, E. Y. Chang, and H. Yamaguchi, Coherent phonon manipulation in coupled mechanical resonators, Nat. Phys. 9, 480 (2013).

[33] T. Faust, J. Rieger, M. J. Seitner, J. P. Kotthaus, and E. M. Weig, Coherent control of a classical nanomechanical two-level system, Nat. Phys. 9, 485 (2013).

[34] I. Mahboob, H. Okamoto, K. Onomitsu, and H. Yamaguchi, Two-Mode Thermal-Noise Squeezing in an Electromechanical Resonator, Phys. Rev. Lett. 113, 167203 (2014).

[35] Y. S. Patil, S. Chakram, L. Chang, and M. Vengalattore, Thermomechanical Two-Mode Squeezing in an Ultrahigh-Q Membrane Resonator, Phys. Rev. Lett. 115, 017202 (2015).

[36] R. Lifshitz and M. Cross, Nonlinear Dynamics of Nanomechanical and Micromechanical Resonators, edited by H. G. Schuster, Reviews of Nonlinear Dynamics and Complexity, Vol. 1 (Wiley-VCH, Weinheim, 2008), pp. 1-52.

[37] N. Shiraishi, K. Funo, and K. Saito, Speed Limit for Classical Stochastic Processes, Phys. Rev. Lett. 121, 070601 (2018).

[38] S. Ito and A. Dechant, Stochastic Time Evolution, Information Geometry, and the Cramér-Rao Bound, Phys. Rev. X 10, 021056 (2020). 